\title{
The diagnosis and management of the Spitz nevus in the pediatric population: a systematic review and meta-analysis protocol
}

\author{
Jean Abboud ${ }^{1}$, Michael Stein ${ }^{1}$, Michele Ramien ${ }^{2}$ and Claudia Malic ${ }^{1,3^{*}}$
}

\begin{abstract}
Background: Spitz nevi are uncommon melanocytic neoplasms found in children. Historically, the diagnosis and management of these tumors has lacked consensus among oncologists, pathologists, plastic surgeons, and dermatologists. Once interpreted and treated as a "juvenile melanoma", many have argued for the benignancy of such tumors in certain patient age groups, encouraging a conservative approach. The lack of consensus surrounding the diagnosis and perceived malignant potential of these tumors has led physicians to approach them on a case-by-case basis and institutional protocols. To date, no evidence-based management guideline exists. The objective of this systematic review is to both collect and appraise the evidence on the diagnosis and management of these tumors.

Methods: A comprehensive electronic literature search will be conducted in PubMed, MEDLINE, Embase, and the Cochrane Library from inception to December 2016. Our search involved collaborating with a healthcare librarian to create a strategy for the OVID/MEDLINE databases. A search of electronic databases for oncology, pathology, plastic surgery, and dermatology abstracts will be performed. Key search terms will include, among several others, "Spitz nevi," "Spitzoid melanoma," "juvenile tumor," and "pediatric". The language of publication will be restricted to English and French. Wherever data allows, meta-analyses will be used to assess differences between Spitz nevi and the tumor of comparison. Additionally, data extraction and summarization using tables will be performed. This review has been registered with PROSPERO (CRD42016034045).
\end{abstract}

Conclusions: This review will systematically and comprehensively review diagnostic and management practices associated with the Spitz nevus. This overview of current literature will hopefully provide the foundation for future standardization of clinical practice.

Systematic review registration: PROSPERO CRD42016034045

Keywords: Spitz nevus, Juvenile melanoma, Diagnosis and management

\section{Background}

Spitz nevi are uncommon melanocytic neoplasms of epithelioid or spindled cells that are found in children [1]. Once treated as a "juvenile melanoma" with malignant potential, these tumors possess a spectrum of clinical and histopathologic presentation that has argued for benignancy [2].

\footnotetext{
* Correspondence: cmalic@cheo.on.ca

'Division of Plastic Surgery, University of Ottawa, 501 Smyth Road, Ottawa, ON K1H 8L6, Canada

${ }^{3}$ Paediatric Plastic Surgeon, Children's Hospital of Eastern Ontario, Ottawa, Canada

Full list of author information is available at the end of the article
}

Historically, the diagnosis and management of Spitz nevi has been fraught with confusion [3]. The lack of consensus surrounding the clinical presentation, histopathologic diagnosis, and perceived biologic potential of these tumors has led plastic surgeons, dermatologists, and pathologists alike to approach these tumors on a case-by-case basis. To date, there is no algorithmic approach for either the diagnosis or the management of these tumors $[4,5]$.

The diagnosis of these tumors is commonly made based on a constellation of clinical and histopathologic features. Spitz nevi are classified as typical or atypical, 
and some use age as a determinant factor for diagnosis and prognosis. Clinically, Spitz nevi are solitary, well-circumscribed papules/nodules located on the face, upper/ lower limb, trunk, or genitalia. The color tends to vary from nonpigmented, red/pink to brown pigmentation. These tumors typically occur in young patients and tend to have a rapid growth phase followed by a static phase. Approximately $50 \%$ of cases occur under the age of 10 , and approximately $70 \%$ are diagnosed in the first two decades of life [6]. They can arise spontaneously or may develop with a preexisting melanocytic nevus. From an epidemiologic standpoint, Spitz nevi are most frequently found in fair-skinned individuals and both sexes are equally affected.

The so-called "atypical Spitz nevus" is a melanocytic proliferation with histopathologic features between a typical Spitz nevus and a Spitzoid melanoma and is associated with an uncertain malignant potential. Few studies have described the dermoscopic features of these tumors [7]. A recent multicenter trial of atypical Spitz nevi documented multicomponent and non-specific dermoscopic patterns and emphasized that these tumors could present in typical spitzoid patterns with amelanotic and hypomelanotic nodules [8].

Pathologic features of Spitz nevi include wellcircumscribed borders, spindled or epithelioid cells, giant nevus cells, uniform nuclear and nucleolar enlargement, and maturation of cells deep within the tumor [9]. More concerning features of malignancy potential are present in an atypical Spitz nevus and include numerous and atypical mitoses, marginal mitoses, lack of Kamino bodies, ulceration, lack of maturation, expansile growth pattern, and diffuse p16 negativity $[9-11]$.

There exists a spectrum in both interdisciplinary and intradisciplinary treatment protocols, which range from monitoring, shave biopsy, punch biopsy, and elliptical excision. An elegant study by Metzger et al. in 2013 illustrated contrasting approaches between plastic surgeons and dermatologists [12]. It also demonstrated that dermatologists were managing Spitz nevi in children on a case-by-case basis and were not adhering to their existing guidelines.

The primary objective of this systematic review is to collect and appraise existing literature on Spitz nevi with the intention of informing future diagnostic and management guidelines. Particular emphasis will be placed on the atypical Spitz nevus subgroup.

To ensure scientific rigor, this systematic review protocol was registered with PROSPERO (CRD42016034045), and was adherent with the Preferred Reporting Items for Systematic Reviews and Meta-Analysis Protocols checklist (PRISMA-P), which can be found in an additional file (Additional file 2).

\section{Methods}

\section{Search strategy}

A comprehensive electronic literature search will be conducted in PubMed, MEDLINE, Embase, and the Cochrane Library from inception to December 2016. Our search will involve collaborating with a healthcare librarian to create a strategy for the OVID/MEDLINE databases. We will do a preliminary scan of oncology, pathology, dermatology, and plastic surgery conference abstracts to determine if a full search of the gray literature is necessary. Key search terms will include, among several others, "Spitz nevi," "Spitzoid melanoma," "juvenile tumor," and "pediatric" (see Additional file 1 for full list). The language of publication will be restricted to the English and French language.

\section{Screening}

We will upload the search results and de-duplicate them using the latest EndNote software. Titles and abstracts will go through a level 1 screening by two independent reviewers based on the inclusion criteria, which can be found in full detail in an additional file (Additional file 3). The selected articles will then go through a level 2 screening by both reviewers by reading the full articles.

\section{Eligibility criteria}

Data on patients 18 years and younger with a clinical diagnosis of Spitz nevi will be included in the study. English and French language studies only will be included. Studies must discuss whether nevi were monitored, and/ or biopsied, and/or excised. We will evaluate the monitoring protocols, the length of time to follow-up, and the timing of subsequent interventions. We will also document the use of dermoscopy and any other specialized evaluation techniques that do not require excision or biopsy. When the tumors of interest are found to be partially biopsied, we will record where possible the method of biopsy (shave biopsy, punch biopsy, incisional biopsy). When the tumor of interest is removed with the intent of complete removal, we will record where possible the method of removal (shave, punch, excision) as well as the margins and the incidence of recurrence.

\section{Data extraction and bias assessment}

This review will be addressing a number of details surrounding the Spitz nevus; therefore, a broad scope of information will be collected from each study. A list of anticipated items is summarized in Table 1. The PRISMA statement will guide the reporting of our findings. The PRISMA-P checklist is available as an additional file (Additional file 2). The subpopulation will be under 12 years of age and 12 to 18 years old. Gender will be compared to search for a predilection of the 
Table 1 Data collection items

\begin{tabular}{|c|c|}
\hline \multirow[t]{9}{*}{ Descriptive details } & Population size \\
\hline & Gender comparison \\
\hline & Age distribution \\
\hline & Size of tumor \\
\hline & Site \\
\hline & Profile \\
\hline & History of change \\
\hline & Family history \\
\hline & History of excessive sun exposure \\
\hline \multirow[t]{24}{*}{ Histopathology } & Cell type \\
\hline & Cohesion \\
\hline & Pigmentation \\
\hline & Mitosis \\
\hline & Giant cell \\
\hline & Epidermal relationship \\
\hline & Dermal characteristics \\
\hline & Clark level \\
\hline & Deep mitosis \\
\hline & Atypical mitosis \\
\hline & Nuclear pleomorphism \\
\hline & High N/C ratio \\
\hline & Asymmetry \\
\hline & Solid growth \\
\hline & Architecture \\
\hline & Peripheral pagetoidism \\
\hline & Apoptosis \\
\hline & Architectural maturation \\
\hline & Desmoplasia \\
\hline & Pushing deep border \\
\hline & Necrosis \\
\hline & Circumscription \\
\hline & Prominent nucleoli \\
\hline & Kamino bodies \\
\hline \multirow[t]{5}{*}{ Management and outcomes } & Excision method \\
\hline & Margins \\
\hline & Death \\
\hline & Sentinel lymph node biopsy and results \\
\hline & Recurrence rate \\
\hline
\end{tabular}

disease for male or female. For relevant studies, bias will be assessed using the Cochrane Collaboration Tool for Assessing Risk of Bias [13] and decisions on exclusion or inclusion may rest on the determined level of bias of a study. Systematic reviews will be assessed using A Measurement Tool to Assess Systematic Reviews (AMSTAR) and will be presented in a table [14]. Non- randomized comparative trials will be assessed using Methodological Index for Non-Randomized Studies (MINORS) [15].

\section{Quality assessment}

Each study that is included will be rated based on the Oxford Centre for Evidence-based Medicine (OCEBM) 2011 Levels of Evidence to allow for recommendations to be drawn from the data [16]. Additionally, the quality of evidence regarding reported outcomes will be assessed using the Grading of Recommendations Assessment, Development and Evaluation (GRADE) system principles [17] and a summary table will be produced.

\section{Statistical analysis}

To address the differentiation between Spitz nevi with malignant tumors or tumors with malignant potential, where appropriate, meta-analyses will be carried out by pooling mean differences and odds ratio (OR). If data from the study is not appropriate for meta-analyses, a narrative presentation or summary will be included. Review Manager 5.3 software package will be used for all meta-analyses tables and calculations.

\section{Heterogeneity}

Due to the complexity of the subject being studied, variability is expected to exist due to factors not limited to measurement error; therefore, a random-effects model (the DerSimonian and Laird method) [18] will be adopted for the meta-analyses with 95\% confidence intervals (CI) and significance at the 5\% level. We will assess heterogeneity between studies using the chisquared test [19], and analysis of the inconsistency index $\left(I^{2}\right)$ will measure how much variation across studies is a result of heterogeneity instead of chance alone (where threshold is I2 > 50\%) [20].

\section{Publication bias}

Where there are sufficient studies included in a forest plot, a funnel plot and test of asymmetry will be used to assess publication bias [21]. Duval and Tweedie trim and fill statistics will be used to adjust for missing studies if publication bias is detected [22-24].

\section{Discussion}

The methodology of this systematic review has been designed to adhere specifically to PRISMA-P protocols in order to be comprehensive and to minimize potential biases. Our objective was to offer a foundation for future diagnosis and management guidelines as comprehensive as possible. 


\section{Limitations}

The highly heterogeneous body of literature on Spitz nevi that is likely to be identified during this search may include publications of low quality with small sample sizes. There is also a potential for selection bias and publication bias due to poor indexing of case series studies. The literature surrounding the diagnosis and management of the Spitz nevus is limited. For this reason, our inclusion criteria were very broad. It included publications on Spitz nevi in the adult population if there was some discussion on the pediatric population. Furthermore, texts from oncology, pathology, dermatology, and plastic surgery were similarly evaluated for a multidisciplinary evaluation of existing literature.

\section{Conclusion}

Information from this meta-analysis will be disseminated broadly via presentation at conferences and publication in peer-reviewed journal in order to ensure our findings have a clinical impact on patients. If an inadequate amount of material is generated to perform a quantifiable metaanalysis, we will conduct a narrative review of the literature and discuss observed trends between specialties.

\section{Additional files}

Additional file 1: "Search Strategy" showing the search terms used for each database search engine and the number of results that the search produced. (DOCX $12 \mathrm{~kb}$ )

Additional file 2: "PRISMA-P checklist" showing the adherence to the PRISMA protocol checklist. (DOC $84 \mathrm{~kb}$ )

Additional file 3: "Inclusion and Exclusion criteria" showing the inclusion and exclusion criteria used when screening the articles. (DOCX $15 \mathrm{~kb}$ )

\section{Abbreviations}

AMSTAR: Assessing the Methodological Quality of Systematic Reviews: GRADE: Grading of Recommendations Assessment, Development and Evaluation; MINORS: Methodological Index for Non-Randomized Studies; PRISMA: Preferred Reporting Items for Systematic Reviews and MetaAnalyses; PRISMA-P: Preferred Reporting Items for Systematic review and Meta-Analysis Protocols

\section{Acknowledgements}

We would like to thank the University of Ottawa librarian Mrs. Karine Fournier for her assistance with developing a search strategy.

\section{Funding}

Not applicable.

\section{Availability of data and materials}

Not applicable.

\section{Authors' contributions}

$\mathrm{CM}$ is the guarantor. All authors contributed equally to the conception and design of the study. MS and JA contributed to the acquisition of data and analysis and interpretation of the results, drafted the manuscript, and provided statistical expertise. All authors contributed to the development of the selection criteria, the risk of bias assessment strategy, and data extraction criteria. The search strategy was developed with the help of JA. CM and MR provided expertise on the diagnosis and management of Spitz nevi. All authors read, provided feedback, and approved the final manuscript.

\section{Competing interests}

The authors declare that they have no competing interests.

\section{Consent for publication}

Not applicable.

Ethics approval and consent to participate

The CHEO Research Ethics Board (REB) has reviewed and approved this study (reference no. 15\180x).

\section{Publisher's Note}

Springer Nature remains neutral with regard to jurisdictional claims in published maps and institutional affiliations.

\section{Author details}

${ }^{1}$ Division of Plastic Surgery, University of Ottawa, 501 Smyth Road, Ottawa, ON K1H 8L6, Canada. ²Division of Dermatology, University of Ottawa, 501 Smyth Road, Ottawa, ON K1H 8L6, Canada. ${ }^{3}$ Paediatric Plastic Surgeon, Children's Hospital of Eastern Ontario, Ottawa, Canada.

Received: 27 November 2016 Accepted: 7 April 2017

Published online: 13 April 2017

References

1. Kernen JA, Ackerman LV. Spindle cell nevi and epithelioid cell nevi (so-called juvenile melanomas) in children and adults: a clinicopathological study of 27 cases. Cancer. 1960;13:612-25.

2. Hafiji J, Rytina E, Burrows NP. The spectrum of spitzoid tumours: a clinical study. Australas J Dermatol. 2012;53(3):211-5.

3. Cerroni $L$, Barnhill $R$, Elder $D$, et al. Melanocytic tumors of uncertain malignant potential — results of a tutorial held at the XXIX Symposium of the International Society of Dermatopathology in Graz. Am J Surg Pathol. 2010;34:314-26.

4. Barnhill RL, Argenyi ZB, From L, et al. Atypical Spitz nevi/tumors: lack of consensus for diagnosis, discrimination from melanoma, and prediction of outcome. Hum Pathol. 1999;30:513-20.

5. Situm M, Bolanca Z, Buljan M, et al. Nevus Spitz-everlasting diagnostic difficulties-the review. Coll Antropol. 2008;32 Suppl 2:171-6.

6. Weedon D, Little JH. Spindle and epithelioid cell nevi in children and adults. A review of 211 cases of the Spitz nevus. Cancer. 1977;40:217-25.

7. Argenziano G, Scalvenzi M, Brunetti $B$, et al. Dermatoscopic pitfalls in differentiating pigmented Spitz naevi from cutaneous melanomas. Br J Dermatol. 1999;141(5):788-93.

8. Moscarella E, Lallas A, Kyrgidis A. Clinical and dermoscopic features of atypical Spitz tumors: a multicenter, retrospective, case-control study. J Am Acad Dermatol. 2015;73(5):777-84.

9. Perez A. Spitz nevus and its variants, distinctive clinical and histopathologic features. Pathol Case Rev. 2007;12(6):235-9.

10. Al Dhaybi R, Agoumi M, Gagne I, et al. p16 expression: a marker of differentiation between childhood malignant melanomas and Spitz nevi. J Am Acad Dermatol. 2011;65(2):357-63.

11. Ferrara G, Cavicchini S, Corradin MT. Hypopigmented atypical Spitzoid neoplasms (atypical Spitz nevi, atypical Spitz tumors, Spitzoid melanoma): a clinicopathological update. Dermatol Pract Concept. 2015;5(1):45-52.

12. Metzger AT, Kane AA, Bayliss SJ. Differences in treatment of Spitz nevi and atypical Spitz tumors in pediatric patients among dermatologists and plastic surgeons. J Am A Dermatol. 2013;149(11):1348-50.

13. Higgins JPT, Altman DG, Gøtzsche PC, et al. The Cochrane Collaboration's tool for assessing risk of bias in randomised trials. British Med J. 2011; 343:d5928.

14. Shea B, Grimshaw J, Wells G, et al. Development of AMSTAR: a measurement tool to assess the methodological quality of systematic reviews. BMC Med Res Meth. 2007:7:10

15. Slim K, Nini E, Forestier D, et al. Methodological Index for Non-Randomized Studies (MINORS): development and validation of a new instrument. ANZ J Surg. 2003;73:712-6.

16. OCEBM Levels of Evidence Working Group. The Oxford 2011 Levels of Evidence. Oxford Centre for Evidence-Based Medicine. 2016. http://www. cebm.net/ocebm-levels-of-evidence/. Accessed 1 Sept 2016.

17. Thomas J, Harden A. Methods for the thematic synthesis of qualitative research in systematic reviews. BMC Med Res Methodol. 2008;8(1):1-10. 
18. DerSimonian R, Laird N. Meta-analysis in clinical trials. Control Clin Trials. 1986;7:177-88

19. Cochran WG. The combination of estimates from different experiments. Biometrics. 1954:10:101-29.

20. Higgins JPT, Thompson SG, Deeks JJ, et al. Measuring inconsistency in meta-analyses. Br Med J. 2003;327(7414):557-60.

21. Egger M, Smith GD, Schneider M, et al. Bias in meta-analysis detected by a simple, graphical test. Br Med J. 1997;315(7109):629-34.

22. Duval SJ, Tweedie RL. Trim and fill: a simple funnel-plot-based method of testing and adjusting for publication bias in meta-analysis. Biometrics. 2000; 56(2):455-63.

23. Duval S, Tweedie RL. A nonparametric "trim and fill" method of accounting for publication bias in meta-analysis. J Am Stat Assoc. 2000;95(449):89-98.

24. Duval SJ. The trim and fill method. In: Rothstein HR, Sutton AJ, Borenstein M, editors. Publication bias in meta-analysis: Prevention, assessment, and adjustments. Chichester: Wiley; 2005. p. 127-44.

Submit your next manuscript to BioMed Central and we will help you at every step:

- We accept pre-submission inquiries

- Our selector tool helps you to find the most relevant journal

- We provide round the clock customer support

- Convenient online submission

- Thorough peer review

- Inclusion in PubMed and all major indexing services

- Maximum visibility for your research

Submit your manuscript at www.biomedcentral.com/submit
Biomed Central 